\title{
Audit of the outcome of peptic ulcer disease diagnosed 10 to 20 years previously
}

\author{
J A Malliwah`, M Tabaqchali, J Watson, C W Venables
}

\begin{abstract}
Methods/Aims-During 1993-1994 an audit of the outcomes of a consecutive series of peptic ulcer patients, first diagnosed endoscopically between 1972-1983, was carried out. Three hundred and thirty six patients fitting the entry criteria were identified, 46 had died in the interval, and 44 were lost to follow up, leaving 246 available for evaluation. All patients completed questionnaires on their current symptomatic state, drug treatment, and details of any operations they had undergone since their original diagnosis. In addition they were asked to indicate, on an analogue scale, their overall assessment of how their ulcer problem was affecting them at the time of the review. Where available hospital records were obtained and analysed for any further admissions and the results of any further endoscopies.
\end{abstract}

Results-Of the 246 patients, 158 were men and 88 female. Duodenal ulcers (DU) were present in 204 and gastric ulcers (GU) in 51 (nine had both a DU and GU). Since the diagnosis 65 patients had undergone surgical treatment: 44 for poor ulcer control, nine for pyloric stenosis, nine for a perforation, one for a major gastrointestinal bleed, and two for a gastric carcinoma developing within two years of the diagnosis of a GU. The overall incidence of ulcer complications during this follow up period (excluding the carcinomas) was $7 \cdot 7 \%$. Initial medical treatment was with histamine $\mathrm{H}_{2}$ blockade in 234 patients $-87 \cdot 4 \%$ cimetidine (C) and $11 \%$ ranitidine $(R)$ - with other agents in the remainder. At follow up 176 patients were still receiving medical treatment (C, $71 \%$ : $R, 22 \%$, other, $7 \%$ ) including 30 who had previously undergone a definitive surgical procedure. Dyspeptic symptoms were recorded in $50.4 \%$ of the patients, abdominal pain being the commonest complaint. There was a significant relation between abdominal pain and the analogue scores provided by the patients with significantly more $(p=0.02)$ of those who had undergone surgical treatment recording this as a continuing problem $(44 \cdot 6 \% v 36 \%)$.

Conclusion-There is no evidence provided by this study that, in these patients, their ulcer disease is undergoing spontaneous remission with time.

(Gut 1996; 38: 812-815)

Keywords: peptic ulcer disease.
There have been many changes in the diagnosis and treatment of peptic ulcer disease since the original studies of Fry ${ }^{1}$ and Greibe et $a l^{2}$ yet there have been no further studies within Europe of the longterm outcome of patients with this condition. At the present time there is considerable interest in the role of Helicobacter pylori eradication therapy on the natural history of this condition and much has been made of the short-term benefit of eradication regimens. However, without a baseline on which to judge the success of such treatments, it will be impossible to discover if this is truly changing the longterm outlook for patients with peptic ulceration or not.

One of the problems with these earlier studies was their reliance on the radiological diagnosis of ulcer disease. With the development of fibreoptic endoscopy, in the early 1970 s, it became possible to directly assess the accuracy of barium meal studies in the diagnosis of active duodenal and gastric ulceration. At the time it was believed that a barium meal, carried out by an experienced radiologist was as accurate as an endoscopic assessment. ${ }^{34}$ However, several studies (including one of our own) showed that as many as $40 \%$ of active duodenal ulcers and up to $50 \%$ of active gastric ulcers may be misdiagnosed by a barium meal. ${ }^{45}$ Radiology often misses superficial mucosal lesions and is particularly inaccurate at distinguishing between a depressed scar and an active ulcer crater. ${ }^{6}$ This makes assessing healing by $x$ ray very difficult. ${ }^{47}$ For these reasons it has to be questioned whether all of the patients included in these earlier studies were suffering from an active ulcer at the time of admission to the study or whether some were already in remission.

A further complicating factor has been the development of an increasing range of effective medical treatments for ulcer disease over the past two decades. This has resulted in a decreased reliance on surgical treatment for controlling relapse in those with severe ulcer disease, as continuous maintenance therapy has proved effective in treating this problem in many patients. ${ }^{89}$ Maintenance therapy has also been claimed to reduce the risk of complications such as haemorrhage and perforation. ${ }^{910}$

For these reasons it is important to reassess what is happening to ulcer disease in the 1990s. This study was designed to help in such an assessment.

\section{Methods}

The records of all patients undergoing upper gastrointestinal endoscopy between 1972 and 
1983 at the Royal Victoria Infirmary and (since August 1981) at the Freeman Hospital in Newcastle upon Tyne were examined. A consecutive series of patients diagnosed as having either an active duodenal ulcer (DU) or gastric ulcer (GU) were identified. Unfortunately, between 1972-4, the results of endoscopies were handwritten on an NCR $x$ ray report form. The top copy was filed directly into the patient's medical record and some endoscopists failed to write on this sufficient detail, such as name, address or record number to allow the subsequent tracing of the patient for follow up. Only those patients where adequate demographic details existed could be used in this study. From 1974 a standardised endoscopy report form was used that overcame this problem!

After diagnosis patients were treated medically, under hospital control, until ulcer healing was endoscopically confirmed. We were one of the first centres to use $\mathrm{H}_{2}$ blockade for ulcer healing (initially with metiamide and later with cimetidine) so most patients included in this study were treated with these drugs initially. Only those where healing proved impossible were subjected to early surgical treatment. After ulcer healing most patients were followed up until their ulcer relapsed. If this occurred rapidly they were then re-treated until the ulcer again healed and were then given maintenance therapy. Thereafter their continued treatment was on a 'shared care' basis under their doctor's direct control with intermittent endoscopies when considered necessary because of symptoms or to assess the need for continued treatment. In the latter case treatment was usually continued provided the patient was well, wished to continue treatment, and was not suffering any side effects from the therapy.

A total of 386 patients were identified who fitted the entry criteria. The hospital records, where available, were recovered and questionnaires sent to the patient's last known address. The questions asked for information on what had happened to them since their original diagnosis, their current symptomatic state, whether they were still taking medical therapy, whether they had had any complications since diagnosis or had undergone surgical treatment. In addition they were provided with a $100 \mathrm{~mm}$ analogue scale on which they were asked to mark how they felt their ulcer problem was controlled at the time of the review (marked excellent at $0 \mathrm{~mm}$ and terrible at $100 \mathrm{~mm}$ ). In addition the hospital records (where available) were analysed for any further endoscopic examinations with their findings, and for any ulcer complications or operative treatment the patient had undergone since the original diagnosis.

The data obtained were recorded on a microcomputer and analysed using the Epi Info Version 5.01B (Centre for Disease Control, Atlanta and World Health Organisation) database. Any differences detected were analysed for significance using the Wilcoxon two sample test at $\mathrm{p}<0 \cdot 05$.

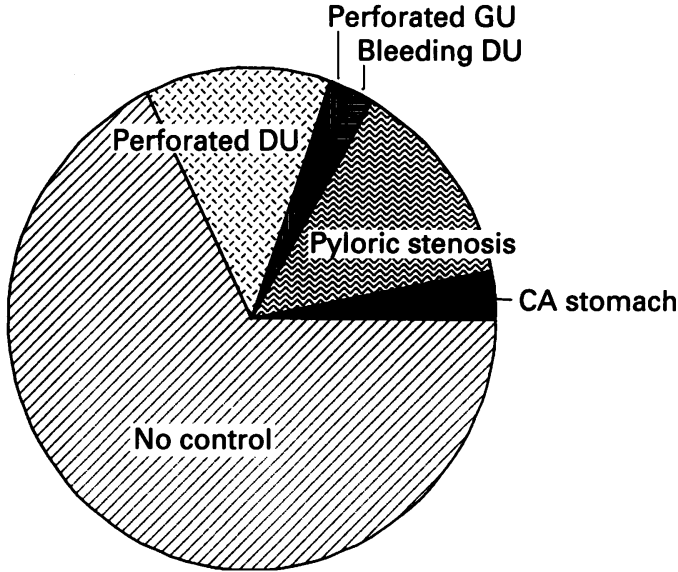

Figure 1: Analysis of the reasons for operation in the 65 patients treated surgically since their original diagnosis.

\section{Results}

A total of 386 patients were initially identified but, on review of the notes, 50 were found to be ineligible as they had undergone previous definitive gastric surgery, 46 (13\%) had died, and $44(12.5 \%)$ could not be traced despite repeated attempts to do so. This left 246 patients who were still alive and available for the study.

Of these 195 had DUs (79\%), 42 GUs (17\%), and nine had both a DU and a GU. Their ages at diagnosis ranged from 15 to 77 years (mean 43.7) and at follow up from 25 to 87 years (mean 55.3). Of the DU group 141 $(69.1 \%)$ were male and 63 female $(39.0 \%)$ : in the GU group $21(41 \cdot 2 \%)$ were male and 30 $(58 \cdot 8 \%)$ female.

Since diagnosis $65(26.4 \%)$ of the patients had undergone surgical treatment: 44 because of inadequate ulcer control, both symptomatically and endoscopically on medical therapy, 19 as a result of an ulcer complication, and two because of carcinoma of the stomach (Fig 1). The carcinomas both occurred within two years of diagnosis in patients originally diagnosed as having a benign GU. The overall complication rate was $7 \cdot 7 \%$ (excluding the carcinomas). Figure 2 shows the timing of subsequent surgical treatment. This shows that most operations were performed between 1981 and 1984 with very few since that time.

Analysis of the original medical treatment provided for their ulcer showed that cimetidine

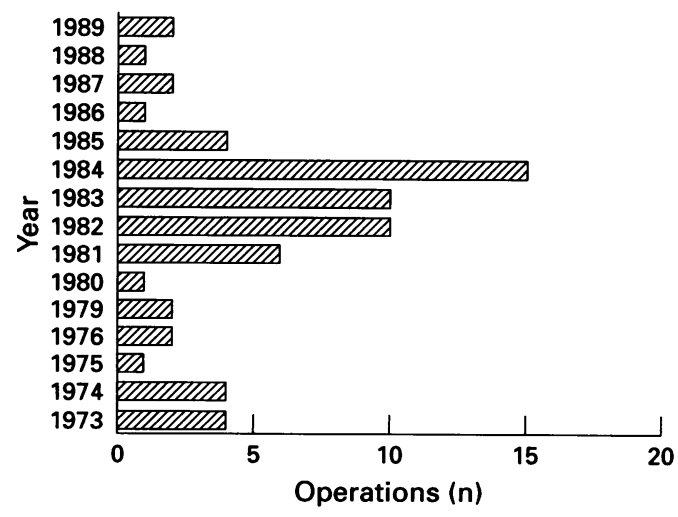

Figure 2: The year in which operation had been performed in the 65 patients treated by operation. 
Figure 3: Analysis of the endoscopic findings at the initial and latest endoscopic assessment in 177 of the patients where adequate hospital records were available.

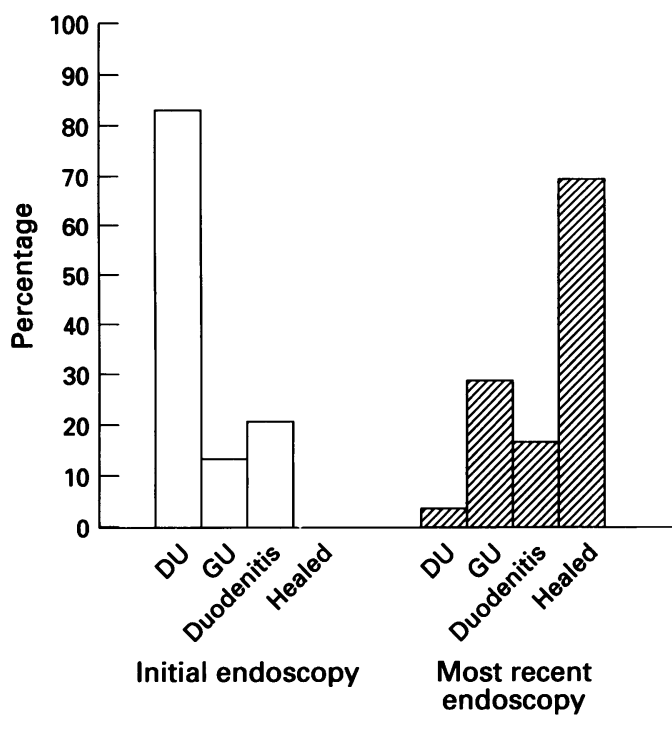

was the drug used in 208 patients $(87 \cdot 4 \%)$ with 26 patients receiving ranitidine $(10.9 \%)$ and the remainder a variety of antacid preparations. At the time of follow up 176 patients $(71.5 \%)$ were still receiving medical therapy, these included 30 patients who had undergone previous surgical treatment designed to 'cure' their ulcer problem $(44.6 \%$ of this second group).

The number and timing of repeat endoscopies in these patients was not standardised, as a result they varied considerably between subjects. For this reason it was only possible to compare the findings between the original examination and those at the most recent examination. There were 177 patients in whom at least one additional endoscopy had been performed (with a mean (SD) interval of $7 \cdot 1(4 \cdot 5)$ years between the examinations). The later examination revealed an active DU in 27, an active $G U$ in seven (two patients having both a DU and GU), active duodenitis in 34, and 140 showed neither an active ulcer or duodenitis (Fig 3).

Analysis of the patient's symptoms at the time of review showed that more than half of the patients still had one or more dyspeptic symptoms, the most common being abdominal
Medically treated ( $n=181)$

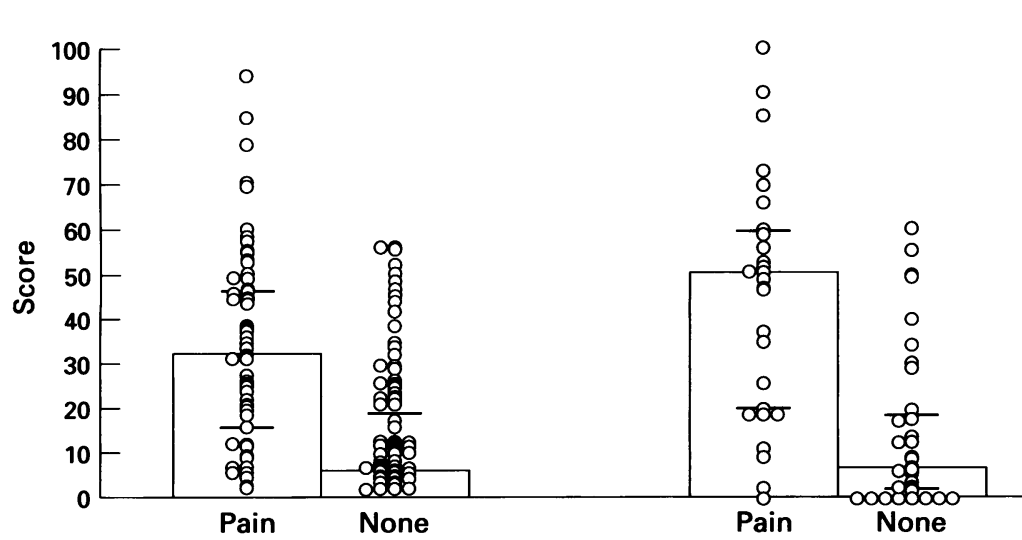

\section{Operated patients} $(n=65)$
Figure 4: Analogue scores (with median \pm quartile) for the patients treated medically throughout compared with those who had been treated surgically, related to whether they said they still had abdominal pain. pain (in $76 \%$ ). Of considerable interest was the fact that 30 of those who had undergone previous surgical treatment $(44 \cdot 6 \%)$ still recorded themselves as having abdominal pain compared with only 67 of those who had been receiving medical treatment throughout this period $(36 \%)(p=N S)$. When the patient's analogue scores were analysed the score given by those who had undergone earlier surgical treatment (median $=18 \cdot 7$ ) was significantly higher $(p=0.043)$ than that given by those still 'receiving medical treatment (median $=12$ ) suggesting that more of the first group felt that their ulcer was still a problem to them! There was also a significant relation $(\mathrm{p}<0.05)$ between the analogue scores and the incidence of abdominal pain in both groups (Fig 4).

\section{Discussion}

This is the first published report of the longterm outcome of peptic ulcer patients where the diagnosis was originally established endoscopically and since the introduction of modern antisecretory drug treatment. While our study is not identical in methodology to those of Fry ${ }^{1}$ and Greibe et $a l^{2}$ it is of some interest to compare our results with theirs. The Danish study was more like ours in that the starting point for their investigation was a hospital $x$ ray diagnosis of peptic ulcer disease while Fry's study was a longitudinal one performed in general practice. It is of interest to note that $26.4 \%$ of our patients (over a mean follow up period of 13.2 years) had undergone surgical treatment despite better medical treatment being available. Most of these operations were performed in the early years after the introduction of $\mathrm{H}_{2}$ blockade, when there was a reluctance to use repeated courses of higher doses of these drugs or to use maintenance treatment for a prolonged period. Since 1984 far fewer operations have been required. It is disappointing to see that so many still required an operation but this may, to some extent, reflect referral to a surgical department with more severe disease less readily controlled medically. Interestingly the number requiring surgery is not dissimilar to that reported by Greibe et al where $22 \%$ of their patients had undergone operation over a mean follow up period of 13 years.

Our group was one of the first to demonstrate, in studies with metiamide between 1971-3, that recurrence of peptic ulceration could be reduced by the use of low dose $\mathrm{H}_{2}$ receptor antagonist given regularly at night. ${ }^{11}$ For this reason we have been keen advocates of this method of treatment for severe peptic ulcer disease and on assessment it was found that most patients included in this longterm follow up had been treated in this way. The finding that the incidence of active peptic ulceration was less at the later follow up endoscopy probably reflects this rather than any change in the severity of the patient's underlying disease. However, 27 patients still showed an active ulcer at this later endoscopy when their symptoms were under control. These asymptomatic ulcers have not been 
associated with any significant problems as only $7 \cdot 7 \%$ of these patients developed a significant peptic ulcer complication during the 13 years of follow up. This compares with $22 \%$ in Fry's $^{1}$ and $20 \%$ in Greibe's ${ }^{2}$ study. This would seem to confirm the effectiveness of maintenance treatment in preventing peptic ulcer complications, as has been reported by other groups. ${ }^{9101213}$

The two gastric carcinomas occurred in patients previously diagnosed as suffering from a benign gastric ulcer. As both occurred within two years of the original diagnosis it seems probable that they were missed at the initial endoscopy (even with routine biopsies) and were not treatment induced. It further emphasises the importance of regular endoscopic follow up of all gastric ulcer patients. Penston and Wormsley ${ }^{14}$ reported that $2 \%$ of their gastric ulcers were subsequently found to be malignant, which compares with an incidence of $3.9 \%$ in this study.

Most of our patients seemed to be satisfied with their symptom control with medical treatment, giving themselves comparatively low analogue scores. It is interesting to note, however, that over $50 \%$ still reported that they had some symptoms present, the commonest being abdominal pain. What was disappointing to find was that those treated surgically still complained of abdominal symptoms and gave themselves significantly higher analogue scores than did those who had been receiving medical treatment throughout. In addition, many were still needing medical treatment. The cause of this persistent pain cannot be established in this study, particularly as we had no control group. However, it is possible that it may be related to the persistence of duodenitis. Kanto and Smith $^{6}$ have suggested that this can cause epigastric pain although our own studies ${ }^{15}$ failed to confirm that duodenitis could be established as a cause of pain.

As a surgical department it is quite possible that the category of patients admitted to this study may be different to those seen in general practice. This might explain the relatively high incidence of surgical treatment used and persistence of pain as our patients are probably selected from those with a more severe form of ulcer disease. In these patients the need to continue with antisecretory treatment and the persistence symptoms after over 13 years suggests that spontaneous remission, as was reported by Fry ${ }^{1}$ and Greibe, ${ }^{2}$ is unlikely to have occurred in our patients. We shall need to wait and see whether eradication of Helicobacter pylori will change the longterm outcome for such patients in the future.

1 Fry J. Peptic ulcer: a profile. BMF 1964; 2: 809-12.

2 Greibe J, Bugge $P$, Gjorup $T$, Lauritzen $T$, Bonnervie $O$, Wulff HR. Long term prognosis of duodenal ulcer; follow up study and survey of doctor's estimates. BMF 1977; 2: 1572-4.

3 Allan RN, Dykes PW, Toye DKM. Diagnostic accuracy of early radiology in acute gastrointestinal haemorrhage. $B M \mathcal{F} 1972 ; 4: 281-4$

4 Forrest JAH, Finlayson NDC, Shearman DJC. Endoscopy in gastrointestinal bleeding. Lancet 1974; ii: 394-7.

5 Kanto RM, Smith FW. Panaendoscopy in the early diagnosis of acute upper gastrointestinal bleeding. Gastroenterology 1973; 65: 728-34.

6 Morrissey JF, Tanaka Y, Thorsen WB. Gastroscopy: a review of the English and Japanese literature. Gastroenterology 1967; 53: 456-76.

7 Kanto RM, Smith FW. Comparison of radiology and duodenoscopy in the diagnosis of duodenal ulcer and duodenitis. Gastroenterology 1972; 62: 769.

8 Venables CW. Criteria for the selection of patients for maintenance therapy. Res Clin Forum 1988; 10: 19-25.

9 Damman HG, Walter TA. Efficiency of continuous therapy for peptic ulcer in controlled clinical trials Aliment Pharmacol Ther 1993; 7 (suppl 2): 17-25.

10 Christensen A, Bousfield R, Christiansen J. Incidence of perforation and bleeding ulcers before and after the introperforation and bleeding ulcers before and after the intro-
duction of $\mathrm{H} 2$ receptor antagonists. Ann Surg 1988; 207: 4-6.

11 Thompson MH, Venables CW, Smith PA, Walker W. Nocturnal metiamide treatment in the management of healed duodenal ulcer. Gut 1977; 18: 438-41.

12 Penston JG, Wormsley KG. Review article: Maintenance treatment with $\mathrm{H} 2$ receptor antagonists for peptic ulcer disease. Aliment Pharmacol Ther 1992; 6: 3-29.

13 Bardhan KD, Hinchcliffe RFC, Bose K, Venables CW, Oliphant CJ, McCarty CF, et al. Six years of continuous cimetidine treatment in peptic ulcer disease. Efficacy and safety. Aliment Pharmacol Ther 1988; 2: 395-405.

14 Penston JG, Wormsley KG. Long-term treatment of gastric ulcers with ranitidine. Aliment Pharmacol Ther 1990; 4: 339-55.

15 Venables CW. Duodenitis. Scand f Gastroenterol 1988; suppl 155: 61-7. 